\title{
Prenatal exposure to non-persistent chemical mixtures and offspring IQ and emotional and behavioral problems
}

Michiel A. van den Dries, Kelly K. Ferguson, Alexander P. Keil, Anjoeka Pronk, Suzanne Spaan, Akhgar Ghassabian, Susana Santos, Vincent W.V. Jaddoe, Leonardo Trasande, Henning Tiemeier, Mònica Guxens

The supplemental material consists of 10 supplemental tables on 12 pages:

Table S1. Descriptive statistics of biomarker concentrations included in the analyses by weeks of gestation (Page S2)

Table S2. Descriptive statistics of biomarker concentrations excluded from the analyses (Page S3)

Table S3 Characteristics of included and excluded participants from the prenatally enrolled Generation R study cohort ( $\mathrm{n}=8879)$ (Page S4)

Table S4. Distributions of averaged chemical biomarker concentrations (ug/g creatinine) across pregnancy $(n=782)$ (Page S5)

Table S5. Pearson and Spearman Rank correlation matrix of pregnancy averaged exposure biomarker concentrations. (Page S6)

Table S6. Descriptive statistics of nonverbal IQ, internalizing problems, attention problems, aggressive behavior, and autistic traits (Page S7)

Table S7. Adjusted estimates for associations between mixtures of pregnancy averaged biomarker concentrations (ug/g creatinine) and nonverbal IQ, emotional and behavioral symptoms at the age of 6 years: model coefficients and differences relative to the lowest quartile of each mixture (Page S8)

Table S8. Adjusted estimates for associations between mixtures of pregnancy averaged biomarker concentrations (ug/g creatinine) and externalizing problems at the age of 6 years: model coefficients and differences relative to the lowest quartile of each mixture (Page S9)

Table S9. Adjusted estimates for associations between mixtures of pregnancy averaged biomarker concentrations (ug/g creatinine) and nonverbal IQ, emotional and behavioral symptoms at the age of 6 years: differences relative to the lowest quartile of each mixture additionally adjusted for birth year (Page S10)

Table S10. Adjusted estimates for associations between mixtures of pregnancy averaged biomarker concentrations (ug/g creatinine) and nonverbal IQ, emotional and behavioral symptoms at the age of 6 years: differences relative to the lowest quartile of each mixture stratified by sex (Page S11-12) 
Table S1. Descriptive statistics of biomarker concentrations included in the analyses by weeks of gestation ${ }^{\text {a }}$

\begin{tabular}{|c|c|c|c|c|c|c|c|c|c|c|}
\hline & Min & $\mathrm{P} 10$ & $\mathrm{P} 20$ & $\mathrm{P} 25$ & P50 & $\mathrm{P} 75$ & P80 & P90 & Max & $\%$ below LOD \\
\hline \multicolumn{11}{|l|}{ Phthalate metabolites } \\
\hline \multicolumn{11}{|l|}{ Monomethyl (MMP) } \\
\hline$<18$ weeks & 0.2 & 1.5 & 2.2 & 2.6 & 5.1 & 9.4 & 10.9 & 17.1 & 505.9 & 0.3 \\
\hline $18-25$ weeks & 0.1 & 1.2 & 1.8 & 2.0 & 3.9 & 7.0 & 8.0 & 12.3 & 2446.9 & 0.1 \\
\hline$>25$ weeks & 0.1 & 1.2 & 1.8 & 2.1 & 4.1 & 8.2 & 9.3 & 15.8 & 1059.8 & 0.5 \\
\hline \multicolumn{11}{|l|}{ Monoethyl (MEP) } \\
\hline$<18$ weeks & 1.3 & 14.3 & 28.9 & 36.3 & 121.1 & 443.5 & 603.8 & 1214.8 & 9482.7 & 0.1 \\
\hline $18-25$ weeks & 1.5 & 10.5 & 23.6 & 29.1 & 85.7 & 274.0 & 362.8 & 725.3 & 10214.4 & 0.0 \\
\hline$>25$ weeks & 1.4 & 17.0 & 32.0 & 40.7 & 123.8 & 407.4 & 534.6 & 1180.9 & 10799.8 & 0.0 \\
\hline \multicolumn{11}{|l|}{ Mono-n-butyl (MnBP) } \\
\hline$<18$ weeks & 0.3 & 3.2 & 5.7 & 7.4 & 17.3 & 33.7 & 40.3 & 64.8 & 2715.6 & 0.6 \\
\hline $18-25$ weeks & 0.2 & 2.9 & 4.3 & 5.2 & 10.4 & 20.5 & 23.9 & 39.3 & 265.9 & 0.0 \\
\hline$>25$ weeks & 0.2 & 2.8 & 4.4 & 5.3 & 10.4 & 20.7 & 25.2 & 42.7 & 2573.4 & 0.3 \\
\hline \multicolumn{11}{|l|}{ Mono-isobutyl (MiBP) } \\
\hline$<18$ weeks & 0.3 & 5.1 & 8.2 & 9.8 & 22.2 & 46.0 & 55.0 & 92.1 & 1826.6 & 0.3 \\
\hline $18-25$ weeks & 0.5 & 2.8 & 4.3 & 5.2 & 10.4 & 20.4 & 24.7 & 43.0 & 1733.0 & 0.0 \\
\hline$>25$ weeks & 0.8 & 4.1 & 6.5 & 7.7 & 13.8 & 30.4 & 37.4 & 70.9 & 2057.1 & 0.1 \\
\hline \multicolumn{11}{|l|}{ Mono-(2-ethyl-5-carboxypentyl) (MECPP) } \\
\hline$<18$ weeks & 0.4 & 4.1 & 6.7 & 8.1 & 15.9 & 30.6 & 36.0 & 60.2 & 906.1 & 0.3 \\
\hline $18-25$ weeks & 0.5 & 2.9 & 4.9 & 5.8 & 10.9 & 20.1 & 23.2 & 35.3 & 93301.2 & 0.1 \\
\hline$>25$ weeks & 0.5 & 5.3 & 7.8 & 9.3 & 17.5 & 33.1 & 41.2 & 65.7 & 451.4 & 0.0 \\
\hline \multicolumn{11}{|l|}{ Mono-(2-ethyl-5-hydroxyhexyl) (MEHHP) } \\
\hline$<18$ weeks & 0.1 & 2.2 & 4.5 & 5.4 & 11.6 & 22.3 & 26.4 & 42.0 & 827.2 & 0.3 \\
\hline $18-25$ weeks & 0.4 & 1.7 & 2.6 & 3.2 & 6.2 & 12.9 & 15.8 & 22.1 & 184.1 & 0.1 \\
\hline$>25$ weeks & 0.1 & 2.5 & 3.9 & 4.5 & 9.6 & 18.9 & 22.2 & 35.7 & 336.9 & 0.3 \\
\hline \multicolumn{11}{|l|}{ Mono-(2-ethyl-5oxohexyl) (MEOHP) } \\
\hline$<18$ weeks & 0.1 & 1.4 & 2.9 & 3.4 & 7.8 & 15.9 & 19.3 & 31.7 & 613.9 & 0.0 \\
\hline $18-25$ weeks & 0.5 & 2.3 & 3.4 & 4.1 & 9.4 & 20.4 & 23.6 & 35.5 & 11831.0 & 0.0 \\
\hline$>25$ weeks & 0.4 & 2.0 & 2.7 & 3.1 & 6.1 & 12.9 & 14.4 & 22.3 & 167.7 & 0.1 \\
\hline \multicolumn{11}{|l|}{ Mono-[(2-carboxymethyl)hexyl] (MCMHP) } \\
\hline$<18$ weeks & 0.3 & 3.9 & 5.9 & 7.0 & 13.4 & 24.8 & 28.4 & 44.1 & 893.9 & 0.1 \\
\hline $18-25$ weeks & 0.1 & 1.2 & 2.1 & 2.4 & 4.4 & 7.8 & 9.0 & 13.8 & 5497.2 & 0.4 \\
\hline$>25$ weeks & 0.1 & 1.0 & 1.6 & 1.9 & 3.6 & 6.6 & 7.6 & 10.7 & 352.0 & 1.2 \\
\hline Mono(3-carboxypropyl) (MCPP) & & & & & & & & & & \\
\hline$<18$ weeks & 0.0 & 0.4 & 0.6 & 0.7 & 1.3 & 2.5 & 2.8 & 4.4 & 120.2 & 0.0 \\
\hline $18-25$ weeks & 0.1 & 0.3 & 0.5 & 0.5 & 0.9 & 1.7 & 2.0 & 3.1 & 63.1 & 0.0 \\
\hline$>25$ weeks & 0.1 & 0.5 & 0.9 & 1.0 & 1.8 & 3.0 & 3.4 & 5.6 & 60.4 & 0.1 \\
\hline Monobenzyl (MBzP) & & & & & & & & & & \\
\hline$<18$ weeks & 0.1 & 1.4 & 2.5 & 3.1 & 6.3 & 12.4 & 14.6 & 21.9 & 554.8 & 13.8 \\
\hline $18-25$ weeks & 0.2 & 1.1 & 1.8 & 2.2 & 5.5 & 10.7 & 13.1 & 25.4 & 522.9 & 0.8 \\
\hline$>25$ weeks & 0.2 & 0.5 & 0.7 & 1.0 & 2.4 & 4.6 & 5.5 & 9.2 & 128.0 & 3.6 \\
\hline Phthalic acid (PA) & & & & & & & & & & \\
\hline$<18$ weeks & 1.3 & 17.2 & 28.8 & 32.8 & 57.5 & 125.4 & 153.6 & 254.3 & 9450.0 & 0.4 \\
\hline $18-25$ weeks & 6.0 & 38.4 & 56.3 & 64.8 & 170.3 & 299.7 & 329.0 & 418.5 & 2189.3 & 0.1 \\
\hline$>25$ weeks & 1.5 & 17.9 & 28.3 & 33.1 & 66.3 & 129.8 & 153.0 & 245.0 & 1770.0 & 0.4 \\
\hline Bisphenols & & & & & & & & & & \\
\hline Bisphenol A (BPA) & & & & & & & & & & \\
\hline$<18$ weeks & 0.1 & 0.4 & 0.6 & 0.7 & 1.7 & 3.5 & 4.3 & 6.8 & 90.2 & 26.0 \\
\hline $18-25$ weeks & 0.2 & 0.5 & 0.7 & 0.9 & 1.7 & 3.4 & 4.0 & 7.2 & 109.5 & 6.7 \\
\hline$>25$ weeks & 0.2 & 0.4 & 0.6 & 0.8 & 1.6 & 3.0 & 3.8 & 6.0 & 64.9 & 13.3 \\
\hline $\begin{array}{l}\text { Dialkylphosphate (DAP) metabolites } \\
\text { dimethyldithiophosphate (DMDTP) }\end{array}$ & & & & & & & & & & \\
\hline$<18$ weeks & 0.1 & 0.2 & 0.2 & 0.2 & 0.5 & 1.1 & 1.4 & 2.8 & 75.6 & 19.9 \\
\hline $18-25$ weeks & 0.1 & 0.2 & 0.2 & 0.3 & 0.5 & 1.1 & 1.5 & 2.6 & 33.5 & 18.2 \\
\hline$>25$ weeks & 0.1 & 0.1 & 0.2 & 0.2 & 0.4 & 1.0 & 1.4 & 2.6 & 38.0 & 17.9 \\
\hline $\begin{array}{l}\text { dimethylthiophosphate (DMTP) } \\
<18 \text { weeks }\end{array}$ & 0.5 & 2.5 & 4.3 & 5.3 & 11.2 & 21.8 & 28.2 & 45.3 & 343.2 & 3.5 \\
\hline $18-25$ weeks & 0.4 & 2.6 & 4.6 & 5.6 & 12.3 & 23.7 & 27.3 & 42.2 & 489.0 & 3.6 \\
\hline$>25$ weeks & 0.4 & 2.7 & 4.5 & 5.6 & 11.3 & 22.1 & 26.3 & 41.7 & 284.9 & 2.4 \\
\hline dimethylphosphate (DMP) & 0.5 & 3.5 & 5.5 & 6.6 & 12.2 & 22.9 & 27.1 & 40.1 & 209.1 & 0.1 \\
\hline $18-25$ weeks & 0.6 & 3.4 & 5.4 & $\begin{array}{l}0.0 \\
6.6\end{array}$ & 12.2 & 21.3 & 24.9 & $\begin{array}{l}40.1 \\
33.7\end{array}$ & 289.3 & $\begin{array}{l}0.1 \\
0.0\end{array}$ \\
\hline$>25$ weeks & 0.6 & 3.4 & 5.2 & 6.1 & 11.5 & 21.2 & 24.1 & 39.6 & 200.3 & 0.0 \\
\hline diethylthiophosphate (DETP) & & & & & & & & & & \\
\hline$<18$ weeks & 0.1 & 0.3 & 0.5 & 0.5 & 1.3 & 2.9 & 3.7 & 6.8 & 117.2 & 12.2 \\
\hline $18-25$ weeks & 0.1 & 0.3 & 0.4 & 0.4 & 1.0 & 2.4 & 2.9 & 5.2 & 99.9 & 11.9 \\
\hline$>25$ weeks & 0.1 & 0.3 & 0.4 & 0.5 & 1.1 & 2.8 & 3.3 & 5.6 & 64.9 & 11.9 \\
\hline Diethylphosphate (DEP) & & & & & & & & & & \\
\hline$<18$ weeks & 0.5 & 1.0 & 1.6 & 1.9 & 3.8 & 7.2 & 8.4 & 13.8 & 1035.7 & 2.7 \\
\hline $18-25$ weeks & 0.5 & 1.0 & 1.5 & 1.8 & 3.5 & 6.6 & 7.8 & 12.4 & 168.5 & 5.4 \\
\hline$>25$ weeks & 0.5 & 1.1 & 1.6 & 1.8 & 3.7 & 7.4 & 8.5 & 13.7 & 77.5 & 4.1 \\
\hline
\end{tabular}

Abbreviations: Min = Minimum, $\mathrm{P}=$ percentile, $\%=$ percentage, $\mathrm{LOD}=$ limit of detection.

a. Descriptive statistics of concentrations for individual biomarkers are in $\mathrm{ng} / \mathrm{mL}$ in which the concentrations below the LOD are excluded. 
Table S2. Descriptive statistics of biomarker concentrations excluded from the analyses ${ }^{\text {a }}$

\begin{tabular}{|c|c|c|c|c|c|c|}
\hline & Min & $\mathrm{P} 25$ & $\mathrm{P} 50$ & $\mathrm{P} 75$ & Max & \% below LOD \\
\hline \multicolumn{7}{|l|}{ Phthalate metabolites } \\
\hline \multicolumn{7}{|l|}{ Mono isononyl (MiNP) } \\
\hline$<18$ weeks & 0.1 & 0.3 & 0.5 & 1.3 & 16.1 & 86.8 \\
\hline $18-25$ weeks & 0.2 & 0.2 & 0.2 & 0.4 & 0.6 & 97.8 \\
\hline$>25$ weeks & - & - & - & - & - & 100.0 \\
\hline \multicolumn{7}{|l|}{ Monocyclohexyl (MCHP) } \\
\hline$<18$ weeks & 0.0 & 0.1 & 0.1 & 0.3 & 6.4 & 85.4 \\
\hline $18-25$ weeks & 0.0 & 0.0 & 0.1 & 0.1 & 1.4 & 91.0 \\
\hline$>25$ weeks & 0.2 & 0.3 & 0.4 & 1.3 & 2.2 & 99.4 \\
\hline \multicolumn{7}{|l|}{ Monooctyl (MOP) } \\
\hline$<18$ weeks & 0.3 & 0.3 & 0.5 & 0.8 & 9.3 & 90.0 \\
\hline $18-25$ weeks & 0.4 & 0.5 & 0.5 & 0.5 & 0.6 & 99.5 \\
\hline$>25$ weeks & 0.3 & 0.4 & 0.4 & 0.7 & 1.7 & 99.1 \\
\hline \multicolumn{7}{|l|}{ Mono-(8-methyl-1-nonyl) (MiDP) } \\
\hline$<18$ weeks & 0.9 & 1.3 & 1.8 & 2.6 & 14.2 & 88.6 \\
\hline $18-25$ weeks & 1.0 & 1.1 & 1.2 & 1.5 & 4.7 & 97.3 \\
\hline$>25$ weeks & 0.9 & 2.3 & 2.9 & 5.1 & 15.1 & 95.9 \\
\hline \multicolumn{7}{|l|}{ Mono-hexyl (MHxP) } \\
\hline $\begin{array}{l}<18 \text { weeks } \\
18-25 \text { weeks }\end{array}$ & 0.0 & 0.2 & 0.3 & 0.6 & 59.9 & 32.8 \\
\hline$>25$ weeks & $\begin{array}{l}0.1 \\
0.0\end{array}$ & $\begin{array}{l}0.1 \\
0.4\end{array}$ & $\begin{array}{l}0.1 \\
0.6\end{array}$ & $\begin{array}{l}0.1 \\
0.7\end{array}$ & $\begin{array}{l}0.4 \\
2.1\end{array}$ & $\begin{array}{l}98.6 \\
97.8\end{array}$ \\
\hline \multicolumn{7}{|l|}{ Mono-2-heptyl (MHpP) } \\
\hline$<18$ weeks & 0.2 & 0.6 & 1.0 & 1.9 & 78.7 & 44.4 \\
\hline $18-25$ weeks & 0.3 & 0.4 & 0.6 & 0.7 & 1.3 & 94.9 \\
\hline$>25$ weeks & 0.3 & 0.7 & 1.0 & 1.7 & 24.5 & 98.2 \\
\hline \multicolumn{7}{|l|}{ Mono-(7-carboxy-n-heptyl) (MCHpP) } \\
\hline$<18$ weeks & 0.1 & 0.1 & 0.1 & 0.1 & 0.2 & 98.6 \\
\hline $18-25$ weeks & - & - & - & - & - & 100.0 \\
\hline$>25$ weeks & 0.3 & 0.9 & 1.5 & 2.6 & 3.7 & 99.6 \\
\hline \multicolumn{7}{|l|}{ Bisphenols } \\
\hline \multicolumn{7}{|l|}{ Bisphenol S } \\
\hline$<18$ weeks & 0.0 & 0.2 & 0.3 & 0.8 & 27.0 & 32.2 \\
\hline $18-25$ weeks & 0.1 & 0.1 & 0.3 & 0.5 & 20.0 & 56.0 \\
\hline$>25$ weeks & 0.1 & 0.3 & 0.6 & 1.4 & 16.7 & 78.2 \\
\hline \multicolumn{7}{|l|}{ Bisphenol F } \\
\hline$<18$ weeks & 0.2 & 0.3 & 0.7 & 1.6 & 82.7 & 65.0 \\
\hline $18-25$ weeks & 0.2 & 0.3 & 0.5 & 1.2 & 14.3 & 85.3 \\
\hline$>25$ weeks & 0.2 & 0.7 & 1.2 & 2.2 & 42.7 & 69.0 \\
\hline \multicolumn{7}{|l|}{ Bisphenol Z } \\
\hline$<18$ weeks & 0.1 & 0.2 & 0.2 & 0.5 & 2.3 & 90.8 \\
\hline $18-25$ weeks & 0.1 & 0.1 & 0.2 & 0.2 & 0.4 & 93.7 \\
\hline$>25$ weeks & 1.0 & 1.0 & 1.1 & 1.2 & 1.3 & 99.7 \\
\hline \multicolumn{7}{|l|}{ Bisphenol B } \\
\hline$<18$ weeks & 0.0 & 0.1 & 0.1 & 0.2 & 1.6 & 85.3 \\
\hline $18-25$ weeks & 0.0 & 0.1 & 0.1 & 0.2 & 0.5 & 96.3 \\
\hline$>25$ weeks & - & - & - & - & - & 100.0 \\
\hline \multicolumn{7}{|l|}{ Bisphenol AP } \\
\hline$<18$ weeks & 0.1 & 0.1 & 0.2 & 0.4 & 2.7 & 87.3 \\
\hline $18-25$ weeks & - & - & - & - & - & 100.0 \\
\hline$>25$ weeks & 0.2 & 0.2 & 0.2 & 0.2 & 0.2 & 99.9 \\
\hline Bisphenol AF & & & & & & \\
\hline$<18$ weeks & - & - & - & - & - & 100.0 \\
\hline $18-25$ weeks & - & - & - & - & - & 100.0 \\
\hline$>25$ weeks & 3.7 & 3.7 & 3.7 & 3.7 & 3.7 & 99.9 \\
\hline Bisphenol P & & & & & & \\
\hline$<18$ weeks & 0.1 & 0.1 & 0.2 & 0.3 & 1.8 & 97.1 \\
\hline $18-25$ weeks & - & - & - & - & - & 100.0 \\
\hline$>25$ weeks & 0.1 & 0.1 & 0.1 & 0.1 & 0.1 & 99.6 \\
\hline Dialkyl phosphate metabolites & & & & & & \\
\hline Diethyldithiophosphate (DEDTP) & & & & & & \\
\hline$<18$ weeks & 0.1 & 0.1 & 0.1 & 0.2 & 34.0 & 81.1 \\
\hline $18-25$ weeks & 0.1 & 0.1 & 0.1 & 0.2 & 5.2 & 84.5 \\
\hline$>25$ weeks & 0.1 & 0.1 & 0.1 & 0.2 & 28.5 & 85.0 \\
\hline
\end{tabular}

Abbreviations: Min $=$ Minimum, $\mathrm{P}=$ percentile, $\%=$ percentage, $\mathrm{LOD}=$ limit of detection.

a. Descriptive statistics of concentrations for individual biomarkers are in $\mathrm{ng} / \mathrm{mL}$ in which the concentrations below the LOD are excluded. 
Table S3. Characteristics of included and excluded participants from the prenatally enrolled Generation R study cohort $(n=8879)$

\begin{tabular}{|c|c|c|c|}
\hline 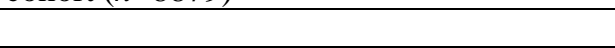 & Distribution $(n=782)^{a}$ & Distribution $(n=8097)^{\mathrm{a}}$ & P-value ${ }^{b}$ \\
\hline Maternal age (years) & $31(28,34)$ & $30(26,33)$ & $<0.001$ \\
\hline Missing, $n$ & - & 2 & \\
\hline Maternal ethnicity & & & $<0.001$ \\
\hline Dutch & 57.7 & 48.0 & \\
\hline Other western & 12.7 & 11.5 & \\
\hline Non-western & 29.6 & 40.5 & \\
\hline Missing, $n$ & - & 501 & \\
\hline Maternal IQ & $100(90,107)$ & $95(84,107)$ & $<0.001$ \\
\hline Missing, $n$ & 19 & 3103 & \\
\hline Maternal education & & & $<0.001$ \\
\hline Low & 14.8 & 28.4 & \\
\hline Intermediate & 30.2 & 31.0 & \\
\hline High & 55.0 & 40.5 & \\
\hline Missing, $n$ & 24 & 794 & \\
\hline Household income & & & $<0.001$ \\
\hline$<1200 € /$ month & 12.6 & 21.7 & \\
\hline $1200-2000 € /$ month & 16.5 & 18.8 & \\
\hline$>2000 € /$ month & 70.9 & 59.4 & \\
\hline Missing, $n$ & 101 & 2134 & \\
\hline Maternal psychopathology & $0.13(0.08,0.33)$ & $0.17(0.08,0.38)$ & $<0.001$ \\
\hline Missing, $n$ & 94 & 2135 & \\
\hline Maternal body mass index $\left(\mathrm{kg} / \mathrm{m}^{2}\right)$ & $22(21,25)$ & $23(21,26)$ & 0.255 \\
\hline Missing, $n$ & 97 & 1652 & \\
\hline Maternal parity & & & $<0.001$ \\
\hline 0 & 62.3 & 54.9 & \\
\hline 1 & 26.6 & 30.2 & \\
\hline$\geq 2$ & 11.1 & 14.9 & \\
\hline Missing, $n$ & 4 & 121 & \\
\hline Maternal marital status & & & $<0.001$ \\
\hline Married/ living with partner & 89.8 & 84.7 & \\
\hline No partner & 10.2 & 15.3 & \\
\hline Missing, $n$ & 29 & 808 & \\
\hline Maternal smoking during pregnancy & & & 0.003 \\
\hline No smoking during pregnancy & 77.2 & 72.3 & \\
\hline Until pregnancy recognized & 8.9 & 8.7 & \\
\hline Continued during pregnancy & 13.9 & 19.0 & \\
\hline Missing, $n$ & 63 & 1076 & \\
\hline $\begin{array}{l}\text { Maternal alcohol consumption during } \\
\text { pregnancy }\end{array}$ & & & $<0.001$ \\
\hline No consumption during pregnancy & 36.7 & 49.2 & \\
\hline Until pregnancy recognized & 17.5 & 12.8 & \\
\hline Continued occasionally & 39.3 & 30.8 & \\
\hline Continued frequently & 6.5 & 7.3 & \\
\hline Missing, $n$ & 40 & 932 & \\
\hline Child sex (female) & 49.1 & 49.6 & 0.785 \\
\hline Missing, $n$ & - & 154 & \\
\hline
\end{tabular}

a. Median $\left(25^{\text {th }}\right.$ percentile, $75^{\text {th }}$ percentile) for continuous variables and percentage for categorical variables.

b. P-value calculated using a chi-squared test for categorical characteristics and an independent $t$-test for continuous characteristics. 
Table S4. Distributions of averaged chemical biomarker concentrations (ug/g creatinine) ${ }^{a}$ across pregnancy $(n=782)$

\begin{tabular}{|c|c|c|c|c|c|}
\hline & Min & $\mathrm{P} 25$ & P50 & P75 & Max \\
\hline \multicolumn{6}{|l|}{ Phthalate metabolites } \\
\hline Monomethyl (MMP) & 0.6 & 2.9 & 4.1 & 6.0 & 175.7 \\
\hline Monoethyl (MEP) & 5.4 & 51.0 & 116.6 & 256.2 & 3022.0 \\
\hline Mono-n-butyl (MnBP) & 1.0 & 7.7 & 12.1 & 18.2 & 103.4 \\
\hline Mono-isobutyl (MiBP) & 1.1 & 9.5 & 14.3 & 24.8 & 170.9 \\
\hline Mono-(2-ethyl-5-carboxypentyl) (MECPP) & 0.6 & 10.0 & 14.5 & 20.9 & 227.3 \\
\hline Mono-(2-ethyl-5-hydroxyhexyl) (MEHHP) & 0.3 & 5.6 & 8.8 & 13.5 & 112.8 \\
\hline Mono-(2-ethyl-5oxohexyl) (MEOHP) & 0.9 & 5.0 & 7.7 & 12.0 & 98.5 \\
\hline Mono-[(2-carboxymethyl)hexyl] (MCMHP) & 0.1 & 4.2 & 5.7 & 7.9 & 105.2 \\
\hline Mono(3-carboxypropyl) (MCPP) & 0.2 & 0.9 & 1.3 & 1.9 & 22.0 \\
\hline Monobenzyl (MBzP) & 0.2 & 1.8 & 3.4 & 6.0 & 67.6 \\
\hline Phthalic acid (PA) & 9.4 & 54.4 & 84.2 & 124.4 & 516.7 \\
\hline \multicolumn{6}{|l|}{ Bisphenols } \\
\hline Bisphenol A (BPA) & 0.1 & 0.7 & 1.1 & 1.8 & 27.6 \\
\hline \multicolumn{6}{|l|}{ Dialkylphosphate (DAP) metabolites } \\
\hline dimethyldithiophosphate (DMDTP) & 0.0 & 0.2 & 0.4 & 0.8 & 8.2 \\
\hline dimethylthiophosphate (DMTP) & 0.0 & 8.9 & 14.8 & 22.4 & 103.0 \\
\hline dimethylphosphate (DMP) & 2.0 & 11.9 & 16.5 & 23.0 & 76.5 \\
\hline diethylthiophosphate (DETP) & 0.0 & 0.5 & 1.2 & 2.1 & 14.2 \\
\hline Diethylphosphate (DEP) & 0.3 & 3.2 & 4.9 & 7.5 & 86.4 \\
\hline
\end{tabular}

Abbreviations: Min = Minimum, $\mathrm{P}=$ percentile, $\mathrm{LOD}=$ limit of detection.

a. Phthalate and bisphenol concentration values below the LOD are imputed using the LOD/sqrt2. Lab reported machine DAP metabolite concentrations below the LOD used. 
Table S5. Pearson and Spearman Rank correlation matrix of pregnancy averaged exposure biomarker concentrations.

\begin{tabular}{|c|c|c|c|c|c|c|c|c|c|c|c|c|c|c|c|c|}
\hline $\begin{array}{l}\begin{array}{l}\text { Pearson } \\
\text { correlations }\end{array} \\
\end{array}$ & MMP & MEP & MCPP & $\mathrm{MiBP}$ & $\mathrm{MnBP}$ & MECPP & MCMHP & $\mathrm{MBzP}$ & PA & MOEHP & MEHHP & BPA & DETP & DEP & DMDTP & DMTP \\
\hline MMP & - & & & & & & & & & & & & & & & \\
\hline MEP & 0.15 & - & & & & & & & & & & & & & & \\
\hline MCPP & 0.22 & 0.03 & - & & & & & & & & & & & & & \\
\hline MiBP & 0.17 & 0.05 & 0.35 & - & & & & & & & & & & & & \\
\hline $\mathrm{MnBP}$ & 0.19 & 0.10 & 0.47 & 0.57 & - & & & & & & & & & & & \\
\hline МЕCРP & 0.24 & 0.03 & 0.52 & 0.39 & 0.42 & - & & & & & & & & & & \\
\hline МСМНР & 0.16 & 0.07 & 0.38 & 0.32 & 0.40 & 0.61 & - & & & & & & & & & \\
\hline $\mathrm{MBzP}$ & 0.15 & 0.14 & 0.22 & 0.33 & 0.37 & 0.26 & 0.30 & - & & & & & & & & \\
\hline PA & 0.38 & 0.55 & 0.34 & 0.25 & 0.29 & 0.35 & 0.30 & 0.17 & - & & & & & & & \\
\hline MEOHP & 0.21 & 0.00 & 0.53 & 0.53 & 0.58 & 0.76 & 0.63 & 0.30 & 0.34 & - & & & & & & \\
\hline МЕНHР & 0.21 & -0.02 & 0.52 & 0.49 & 0.56 & 0.74 & 0.65 & 0.24 & 0.31 & 0.90 & - & & & & & \\
\hline BPA & 0.05 & 0.01 & 0.18 & 0.11 & 0.14 & 0.25 & 0.14 & 0.08 & 0.24 & 0.20 & 0.19 & - & & & & \\
\hline DETP & 0.06 & -0.06 & 0.05 & -0.02 & -0.02 & 0.08 & 0.04 & -0.10 & 0.00 & 0.07 & 0.08 & 0.09 & - & & & \\
\hline DEP & 0.09 & -0.05 & 0.00 & -0.10 & -0.01 & 0.00 & 0.05 & -0.05 & -0.02 & 0.04 & 0.06 & -0.05 & 0.40 & - & & \\
\hline DMDTP & 0.01 & -0.05 & 0.04 & -0.06 & -0.05 & 0.10 & -0.02 & -0.05 & -0.04 & 0.04 & 0.05 & 0.11 & 0.53 & 0.16 & - & \\
\hline DMTP & 0.03 & -0.08 & 0.12 & 0.03 & 0.01 & 0.16 & 0.01 & -0.03 & 0.00 & 0.14 & 0.14 & 0.07 & 0.63 & 0.22 & 0.70 & - \\
\hline DMP & 0.09 & -0.06 & 0.11 & 0.03 & 0.05 & 0.11 & 0.09 & 0.01 & 0.01 & 0.14 & 0.17 & -0.05 & 0.25 & 0.60 & 0.25 & 0.36 \\
\hline $\begin{array}{l}\text { Spearman } \\
\text { Rank } \\
\text { correlations } \\
\end{array}$ & MMP & MEP & $\mathrm{MCPP}$ & $\mathrm{MiBP}$ & $\mathrm{MnBP}$ & MECPP & МСMHP & $\mathrm{MBzP}$ & PA & MOEHP & MEHHP & BPA & DETP & DEP & DMDTP & DMTP \\
\hline MMP & - & & & & & & & & & & & & & & & \\
\hline MEP & 0.18 & - & & & & & & & & & & & & & & \\
\hline MCPP & 0.29 & 0.03 & - & & & & & & & & & & & & & \\
\hline MiBP & 0.24 & 0.07 & 0.38 & - & & & & & & & & & & & & \\
\hline $\mathrm{MnBP}$ & 0.25 & 0.09 & 0.48 & 0.62 & - & & & & & & & & & & & \\
\hline МЕCРP & 0.30 & 0.00 & 0.55 & 0.41 & 0.43 & - & & & & & & & & & & \\
\hline МСМHР & 0.22 & 0.06 & 0.39 & 0.36 & 0.41 & 0.59 & - & & & & & & & & & \\
\hline $\mathrm{MBzP}$ & 0.19 & 0.12 & 0.22 & 0.35 & 0.39 & 0.25 & 0.31 & - & & & & & & & & \\
\hline PA & 0.38 & 0.54 & 0.33 & 0.26 & 0.29 & 0.34 & 0.30 & 0.15 & - & & & & & & & \\
\hline MEOHP & 0.29 & -0.02 & 0.54 & 0.54 & 0.57 & 0.77 & 0.63 & 0.30 & 0.32 & - & & & & & & \\
\hline MEHHP & 0.28 & -0.04 & 0.53 & 0.51 & 0.56 & 0.75 & 0.64 & 0.25 & 0.29 & 0.92 & - & & & & & \\
\hline BPA & 0.06 & 0.01 & 0.17 & 0.10 & 0.13 & 0.25 & 0.13 & 0.05 & 0.24 & 0.21 & 0.20 & - & & & & \\
\hline DETP & 0.09 & -0.07 & 0.08 & -0.02 & 0.00 & 0.12 & 0.07 & -0.10 & 0.03 & 0.11 & 0.13 & 0.07 & - & & & \\
\hline DEP & 0.12 & -0.04 & 0.02 & -0.08 & 0.00 & 0.02 & 0.07 & -0.03 & 0.02 & 0.05 & 0.07 & -0.04 & 0.47 & - & & \\
\hline DMDTP & 0.02 & -0.04 & 0.03 & -0.07 & -0.05 & 0.12 & 0.00 & -0.03 & -0.02 & 0.05 & 0.05 & 0.10 & 0.49 & 0.19 & - & \\
\hline DMTP & 0.06 & -0.10 & 0.16 & 0.04 & 0.02 & 0.18 & 0.04 & -0.05 & 0.01 & 0.17 & 0.17 & 0.06 & 0.61 & 0.34 & 0.72 & - \\
\hline DMP & 0.10 & -0.08 & 0.11 & 0.03 & 0.06 & 0.09 & 0.11 & 0.03 & 0.00 & 0.12 & 0.15 & -0.05 & 0.31 & 0.61 & 0.29 & 0.52 \\
\hline
\end{tabular}

See Table S1 for biomarker abbreviations and Figure 1 for a graphical presentation of the Pearson correlation matrix. 
Table S6. Descriptive statistics of nonverbal IQ, internalizing problems, attention problems, aggressive behavior, and autistic traits

\begin{tabular}{lcc}
\hline & Mean & SD \\
\hline Nonverbal IQ (N=708) & 102.3 & 15.4 \\
Internalizing problems $(\mathrm{N}=773)$ & 6.0 & 5.7 \\
Attention problems $(\mathrm{N}=775)$ & 1.6 & 1.8 \\
Aggressive behavior $(\mathrm{N}=771)$ & 6.3 & 5.6 \\
Autistic traits $(\mathrm{N}=662)$ & 0.2 & 0.2 \\
\hline
\end{tabular}


Table S7. Adjusted ${ }^{a}$ estimates for associations between mixtures of pregnancy averaged biomarker concentrations (ug/g creatinine) and nonverbal IQ, emotional and behavioral symptoms at the age of 6 years: model coefficients and differences relative to the lowest quartile of each mixture

\begin{tabular}{|c|c|c|c|c|c|c|c|c|c|c|}
\hline & \multicolumn{2}{|c|}{$\begin{array}{l}\text { Nonverbal } \\
\text { IQ }(n=708)\end{array}$} & \multicolumn{2}{|c|}{$\begin{array}{c}\text { Internalizing } \\
\text { problems }(n=773)^{\mathrm{b}}\end{array}$} & \multicolumn{2}{|c|}{$\begin{array}{c}\text { Attention } \\
\text { problems }(n=775)^{\mathrm{b}}\end{array}$} & \multicolumn{2}{|c|}{$\begin{array}{c}\text { Aggressive behavior } \\
(n=771)^{\mathrm{b}}\end{array}$} & \multicolumn{2}{|c|}{$\begin{array}{c}\text { Autistic traits } \\
(n=622)^{\mathrm{b}}\end{array}$} \\
\hline & $\mathrm{B}$ & $95 \% \mathrm{CI}$ & $\mathrm{B}$ & $95 \% \mathrm{CI}$ & $\mathrm{B}$ & $95 \% \mathrm{CI}$ & $\mathrm{B}$ & $95 \% \mathrm{CI}$ & $\mathrm{B}$ & $95 \% \mathrm{CI}$ \\
\hline \multicolumn{11}{|l|}{ Overall } \\
\hline Mixture & -5.23 & $-9.07,-1.40$ & 0.25 & $-0.09,0.58$ & 0.12 & $-0.06,0.31$ & 0.31 & $-0.02,0.63$ & -0.02 & $-0.06,0.01$ \\
\hline$\wedge 2$ & 1.23 & $0.18,2.27$ & -0.08 & $-0.16,0.00$ & -0.06 & $-0.11,0.00$ & -0.11 & $-0.20,-0.02$ & - & - \\
\hline \multicolumn{11}{|l|}{ Difference per quartile } \\
\hline Q1-Q2 & -4.00 & $-7.00,-1.00$ & 0.17 & $-0.10,0.44$ & 0.06 & $-0.08,0.21$ & 0.20 & $-0.06,0.46$ & -0.02 & $-0.06,0.02$ \\
\hline Q1-Q3 & -5.54 & $-10.24,-0.85$ & 0.18 & $-0.26,0.61$ & 0.02 & $-0.22,0.26$ & 0.17 & $-0.23,0.58$ & -0.04 & $-0.12,0.03$ \\
\hline Q1-Q4 & -4.63 & $-10.76,1.51$ & 0.02 & $-0.54,0.59$ & -0.14 & $-0.49,0.21$ & -0.07 & $-0.61,0.46$ & -0.06 & $-0.18,0.05$ \\
\hline \multicolumn{11}{|l|}{ Phthalate metabolites ${ }^{c}$} \\
\hline Mixture & -4.68 & $-8.44,-0.91$ & -0.02 & $-0.15,0.11$ & 0.17 & $0.00,0.35$ & 0.30 & $0.01,0.59$ & -0.02 & $-0.04,0.01$ \\
\hline$\wedge 2$ & 1.24 & $0.19,2.28$ & - & - & -0.06 & $-0.11,0.00$ & -0.11 & $-0.20,-0.02$ & - & - \\
\hline \multicolumn{11}{|l|}{ Difference per quartile } \\
\hline $\mathrm{Q} 1-\mathrm{Q} 2$ & -3.44 & $-6.33,-0.55$ & -0.02 & $-0.15,0.11$ & 0.12 & $-0.01,0.25$ & 0.19 & $-0.02,0.41$ & -0.02 & $-0.04,0.01$ \\
\hline Q1-Q3 & -4.41 & $-8.76,-0.05$ & -0.04 & $-0.31,0.22$ & 0.13 & $-0.07,0.32$ & 0.17 & $-0.14,0.47$ & -0.03 & $-0.09,0.02$ \\
\hline Q1-Q4 & -2.90 & $-8.35,2.55$ & -0.06 & $-0.46,0.34$ & 0.02 & $-0.23,0.27$ & -0.08 & $-0.45,0.29$ & -0.05 & $-0.13,0.03$ \\
\hline \multicolumn{11}{|l|}{ Bisphenol $^{\mathrm{d}}$} \\
\hline Mixture & 0.42 & $-0.60,1.45$ & -0.01 & $-0.08,0.07$ & -0.04 & $-0.09,0.01$ & -0.04 & $-0.12,0.04$ & 0.00 & $-0.02,0.01$ \\
\hline$\wedge 2$ & - & - & - & - & - & - & - & - & - & - \\
\hline \multicolumn{11}{|l|}{ Difference per quartile } \\
\hline Q1-Q2 & 0.42 & $-0.63,1.47$ & -0.01 & $-0.08,0.07$ & -0.04 & $-0.09,0.01$ & -0.04 & $-0.11,0.03$ & 0.00 & $-0.02,0.01$ \\
\hline Q1-Q3 & 0.84 & $-1.26,2.95$ & -0.01 & $-0.17,0.14$ & -0.08 & $-0.19,0.02$ & -0.08 & $-0.22,0.06$ & -0.01 & $-0.04,0.02$ \\
\hline Q1-Q4 & 1.26 & $-1.89,4.42$ & -0.02 & $-0.25,0.21$ & -0.13 & $-0.28,0.03$ & -0.12 & $-0.33,0.10$ & -0.01 & $-0.06,0.04$ \\
\hline \multicolumn{11}{|l|}{$\begin{array}{l}\text { OP pesticide } \\
\text { metabolites }\end{array}$} \\
\hline Mixture & -0.99 & $-2.44,0.46$ & 0.26 & $-0.02,0.55$ & -0.03 & $-0.1,0.04$ & 0.03 & $-0.07,0.14$ & 0.00 & $-0.02,0.02$ \\
\hline$\wedge 2$ & - & - & -0.08 & $-0.16,0.01$ & - & - & - & - & - & - \\
\hline \multicolumn{11}{|l|}{ Difference per quartile } \\
\hline $\mathrm{Q} 1-\mathrm{Q} 2$ & -0.99 & $-2.27,0.29$ & 0.19 & $-0.02,0.39$ & -0.03 & $-0.11,0.04$ & 0.03 & $-0.08,0.14$ & 0.00 & $-0.03,0.02$ \\
\hline Q1-Q3 & -1.98 & $-4.55,0.58$ & 0.22 & $-0.07,0.50$ & -0.06 & $-0.22,0.09$ & 0.07 & $-0.15,0.28$ & -0.01 & $-0.05,0.04$ \\
\hline Q1-Q4 & -2.97 & $-6.82,0.88$ & 0.09 & $-0.23,0.42$ & -0.10 & $-0.32,0.13$ & 0.10 & $-0.23,0.43$ & -0.01 & $-0.08,0.06$ \\
\hline
\end{tabular}

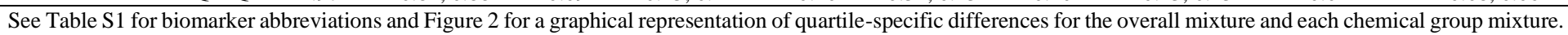

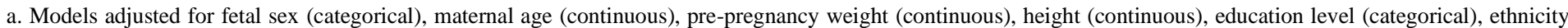

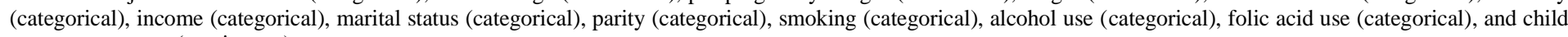
age at assessment (continuous).

b. Square root transformed and additionally adjusted for maternal psychopathology.

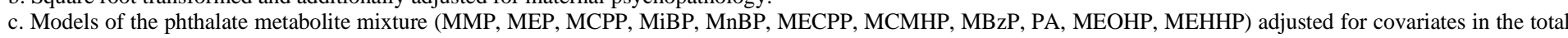
mixtures model plus log 10-transformed pregnancy-averaged concentrations of bisphenol A (BPA) and OP pesticide metabolites (DMDTP, DMTP, DMP, DETP, DEP).

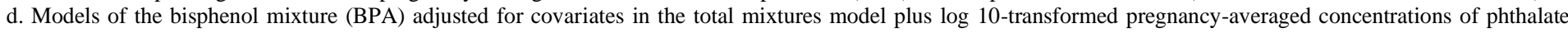
(MMP, MEP, MCPP, MiBP, MnBP, MECPP, MCMHP, MBzP, PA, MEOHP, MEHHP) and OP pesticide metabolites (DMDTP, DMTP, DMP, DETP, DEP).

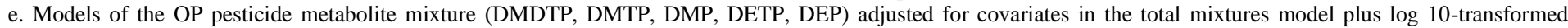
pregnancy-averaged concentrations of phthalate metabolites (MMP, MEP, MCPP, MiBP, MnBP, MECPP, MCMHP, MBzP, PA, MEOHP, MEHHP) and bisphenol A.

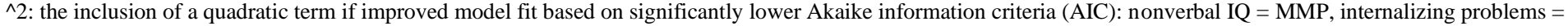
DETP, attention problems $=$ MEHHP, aggressive behavior $=$ MCPP. 
Table S8. Adjusted a estimates for associations between mixtures of pregnancy averaged biomarker concentrations (ug/g creatinine) and externalizing problems at the age of 6 years: model coefficients and differences relative to the lowest quartile of each mixture

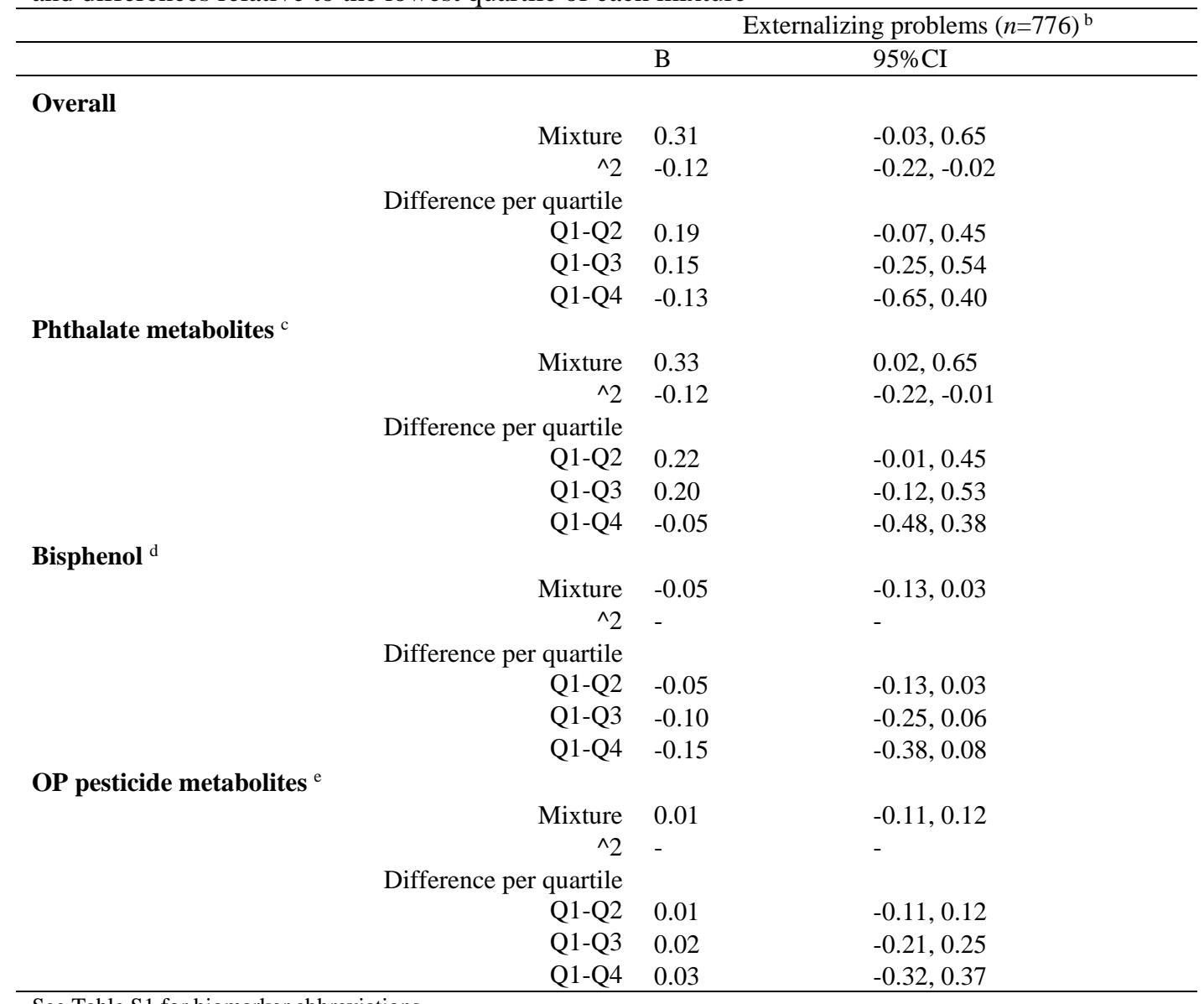

See Table S1 for biomarker abbreviations.

a. Models adjusted for fetal sex (categorical), maternal age (continuous), pre-pregnancy weight (continuous), height (continuous), education level (categorical), ethnicity (categorical), income (categorical), marital status (categorical), parity (categorical), smoking (categorical), alcohol use (categorical), folic acid use (categorical), and child age at assessment (continuous).

b. Square root transformed and additionally adjusted for maternal psychopathology.

c. Models of the phthalate metabolite mixture (MMP, MEP, MCPP, MiBP, MnBP, MECPP, MCMHP, MBzP, PA, MEOHP, MEHHP) adjusted for covariates in the total mixtures model plus log 10-transformed pregnancy-averaged concentrations of bisphenol A (BPA) and OP pesticide metabolites (DMDTP, DMTP, DMP, DETP, DEP).

d. Models of the bisphenol mixture (BPA) adjusted for covariates in the total mixtures model plus log 10-transformed pregnancy-averaged concentrations of phthalate (MMP, MEP, MCPP, MiBP, MnBP, MECPP, MCMHP, MBzP, PA, MEOHP, MEHHP) and OP pesticide metabolites (DMDTP, DMTP, DMP, DETP, DEP).

e. Models of the OP pesticide metabolite mixture (DMDTP, DMTP, DMP, DETP, DEP) adjusted for covariates in the total mixtures model plus log 10-transformed pregnancy-averaged concentrations of phthalate metabolites (MMP, MEP, MCPP, MiBP, MnBP, MECPP, MCMHP, MBzP, PA, MEOHP, MEHHP) and bisphenol A.

^2: the inclusion of a quadratic term if improved model fit based on significantly lower Akaike information criteria (AIC): Externalizing problems $=$ MCPP. 
Table S9. Adjusted ${ }^{a}$ estimates for associations between mixtures of pregnancy averaged biomarker concentrations (ug/g creatinine) and nonverbal IQ, emotional and behavioral symptoms at the age of 6 years: differences relative to the lowest quartile of each mixture additionally adjusted for birth year

\begin{tabular}{|c|c|c|c|c|c|c|c|c|c|c|}
\hline & \multicolumn{2}{|c|}{$\begin{array}{l}\text { Nonverbal } \\
\text { IQ }(n=708)\end{array}$} & \multicolumn{2}{|c|}{$\begin{array}{c}\text { Internalizing } \\
\text { problems }(n=773) \mathrm{b}\end{array}$} & \multicolumn{2}{|c|}{$\begin{array}{c}\text { Attention } \\
\text { problems }(n=775)^{b}\end{array}$} & \multicolumn{2}{|c|}{$\begin{array}{l}\text { Aggressive behavior } \\
\quad(n=771)^{\mathrm{b}}\end{array}$} & \multicolumn{2}{|c|}{$\begin{array}{c}\text { Autistic traits } \\
(n=622)^{\mathrm{b}}\end{array}$} \\
\hline & $\mathrm{B}$ & $95 \% \mathrm{CI}$ & $\mathrm{B}$ & $95 \% \mathrm{CI}$ & $\mathrm{B}$ & $95 \% \mathrm{CI}$ & $\mathrm{B}$ & $95 \% \mathrm{CI}$ & $\mathrm{B}$ & $95 \% \mathrm{CI}$ \\
\hline \multicolumn{11}{|l|}{ Overall } \\
\hline \multicolumn{11}{|l|}{ Difference per quartile } \\
\hline Q1-Q2 & -4.00 & $-7.00,-1.00$ & 0.17 & $-0.10,0.45$ & 0.08 & $-0.06,0.22$ & 0.20 & $-0.06,0.46$ & -0.02 & $-0.06,0.02$ \\
\hline Q1-Q3 & -5.43 & $-10.13,-0.74$ & 0.18 & $-0.26,0.62$ & 0.03 & $-0.21,0.27$ & 0.18 & $-0.23,0.58$ & -0.04 & $-0.12,0.03$ \\
\hline Q1-Q4 & -4.30 & $-10.44,1.84$ & 0.03 & $-0.54,0.60$ & -0.14 & $-0.49,0.21$ & -0.06 & $-0.59,0.46$ & -0.06 & $-0.18,0.05$ \\
\hline \multicolumn{11}{|l|}{ Phthalate metabolites ${ }^{c}$} \\
\hline \multicolumn{11}{|l|}{ Difference per quartile } \\
\hline Q1-Q2 & -3.53 & $-6.43,-0.62$ & -0.02 & $-0.15,0.11$ & 0.14 & $0.01,0.27$ & 0.20 & $-0.02,0.41$ & -0.02 & $-0.04,0.01$ \\
\hline Q1-Q3 & -4.47 & $-8.85,-0.09$ & -0.04 & $-0.31,0.22$ & 0.15 & $-0.04,0.34$ & 0.17 & $-0.14,0.48$ & -0.03 & $-0.09,0.02$ \\
\hline Q1-Q4 & -2.82 & $-8.30,2.66$ & -0.06 & $-0.46,0.34$ & 0.04 & $-0.21,0.29$ & -0.08 & $-0.45,0.30$ & -0.05 & $-0.13,0.03$ \\
\hline \multicolumn{11}{|l|}{ Bisphenol $^{\mathrm{d}}$} \\
\hline \multicolumn{11}{|l|}{ Difference per quartile } \\
\hline Q1-Q2 & 0.42 & $-0.64,1.47$ & -0.01 & $-0.08,0.07$ & -0.04 & $-0.09,0.01$ & -0.04 & $-0.11,0.03$ & 0.00 & $-0.02,0.01$ \\
\hline Q1-Q3 & 0.84 & $-1.28,2.95$ & -0.02 & $-0.17,0.14$ & -0.08 & $-0.19,0.02$ & -0.08 & $-0.22,0.06$ & -0.01 & $-0.04,0.02$ \\
\hline Q1-Q4 & 1.25 & $-1.91,4.42$ & -0.02 & $-0.25,0.21$ & -0.12 & $-0.28,0.03$ & -0.12 & $-0.33,0.10$ & -0.01 & $-0.06,0.04$ \\
\hline \multicolumn{11}{|l|}{$\begin{array}{l}\text { OP pesticide } \\
\text { metabolites }\end{array}$} \\
\hline \multicolumn{11}{|l|}{ Difference per quartile } \\
\hline Q1-Q2 & -0.90 & $-2.19,0.39$ & 0.19 & $-0.01,0.40$ & -0.04 & $-0.12,0.04$ & 0.03 & $-0.07,0.14$ & 0.00 & $-0.03,0.02$ \\
\hline Q1-Q3 & -1.80 & $-4.38,0.78$ & 0.23 & $-0.06,0.51$ & -0.08 & $-0.23,0.07$ & 0.07 & $-0.15,0.29$ & -0.01 & $-0.05,0.04$ \\
\hline Q1-Q4 & -2.70 & $-6.57,1.17$ & 0.10 & $-0.22,0.43$ & -0.12 & $-0.35,0.11$ & 0.10 & $-0.22,0.43$ & -0.01 & $-0.08,0.06$ \\
\hline
\end{tabular}

See Table $\mathrm{S} 1$ for biomarker abbreviations.

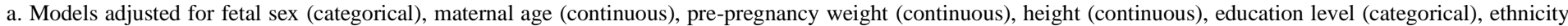

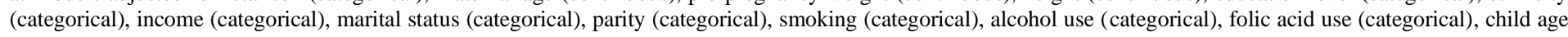
at assessment (continuous), and birth year (continuous).

b. Square root transformed and additionally adjusted for maternal psychopathology.

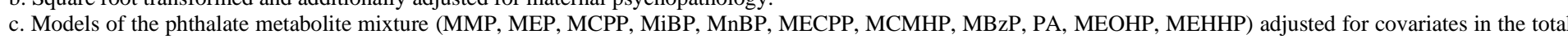
mixtures model plus log 10-transformed pregnancy-averaged concentrations of bisphenol A (BPA) and OP pesticide metabolites (DMDTP, DMTP, DMP, DETP, DEP).

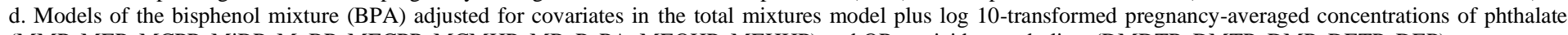
(MMP, MEP, MCPP, MiBP, MnBP, MECPP, MCMHP, MBzP, PA, MEOHP, MEHHP) and OP pesticide metabolites (DMDTP, DMTP, DMP, DETP, DEP).

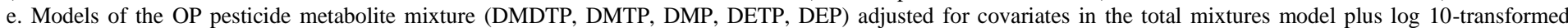
pregnancy-averaged concentrations of phthalate metabolites (MMP, MEP, MCPP, MiBP, MnBP, MECPP, MCMHP, MBzP, PA, MEOHP, MEHHP) and bisphenol A.

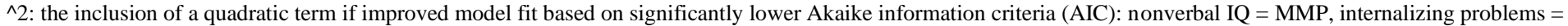
DETP, attention problems = MEHHP, aggressive behavior $=$ MCPP. 
Table S10. Adjusted ${ }^{\mathrm{a}, \mathrm{b}}$ estimates for associations between mixtures of pregnancy averaged biomarker concentrations (ug/g creatinine) and nonverbal IQ, emotional and behavioral symptoms at the age of 6 years: differences relative to the lowest quartile of each mixture stratified by sex

\begin{tabular}{|c|c|c|c|c|c|c|c|c|c|c|c|}
\hline & & \multicolumn{2}{|c|}{$\begin{array}{c}\text { Nonverbal } \\
\text { IQ }(n=708)^{\mathrm{c}}\end{array}$} & \multicolumn{2}{|c|}{$\begin{array}{c}\text { Internalizing } \\
\text { problems }(n=773)^{\mathrm{c}, \mathrm{d}}\end{array}$} & \multicolumn{2}{|c|}{$\begin{array}{c}\text { Attention } \\
\text { problems }(n=775)^{\mathrm{c}, \mathrm{d}}\end{array}$} & \multicolumn{2}{|c|}{$\begin{array}{c}\text { Aggressive behavior } \\
(n=771)^{\mathrm{c}, \mathrm{d}}\end{array}$} & \multicolumn{2}{|c|}{$\begin{array}{c}\text { Autistic } \\
\text { traits }(n=622)^{\mathrm{d}}\end{array}$} \\
\hline & & $\mathrm{B}$ & $95 \% \mathrm{CI}$ & $\mathrm{B}$ & $95 \% \mathrm{CI}$ & $\mathrm{B}$ & $95 \% \mathrm{CI}$ & $\mathrm{B}$ & $95 \% \mathrm{CI}$ & $\mathrm{B}$ & $95 \% \mathrm{CI}$ \\
\hline \multicolumn{12}{|l|}{ All classes } \\
\hline & Q1-Q2 & -4.00 & $-7.00,-1.00$ & .0 .17 & $-0.10,0.44$ & 0.06 & $-0.08,0.21$ & 0.20 & $-0.06,0.46$ & -0.02 & $-0.06,0.02$ \\
\hline & Q1-Q3 & -5.54 & $-10.24,-0.85$ & 0.18 & $-0.26,0.61$ & 0.02 & $-0.22,0.26$ & 0.17 & $-0.23,0.58$ & -0.04 & $-0.12,0.03$ \\
\hline & Q1-Q4 & -4.63 & $-10.76,1.51$ & 0.02 & $-0.54,0.59$ & -0.14 & $-0.49,0.21$ & -0.07 & $-0.61,0.46$ & -0.06 & $-0.18,0.05$ \\
\hline \multicolumn{12}{|l|}{ Boys } \\
\hline & Q1-Q2 & -4.58 & $-9.66,0.49$ & 0.05 & $-0.32,0.41$ & -0.01 & $-0.27,0.26$ & 0.03 & $-0.35,0.41$ & -0.04 & $-0.09,0.01$ \\
\hline & Q1-Q3 & -5.32 & $-13.11,2.48$ & -0.02 & $-0.60,0.56$ & -0.11 & $-0.54,0.32$ & -0.18 & $-0.78,0.41$ & -0.07 & $-0.17,0.03$ \\
\hline & Q1-Q4 & -2.20 & $-11.98,7.59$ & -0.21 & $-0.98,0.57$ & -0.31 & $-0.88,0.25$ & -0.64 & $-1.44,0.15$ & -0.11 & $-0.26,0.04$ \\
\hline \multicolumn{12}{|l|}{ Girls } \\
\hline & Q1-Q2 & -4.61 & $-8.63,-0.58$ & 0.25 & $-0.11,0.62$ & 0.18 & $-0.05,0.41$ & 0.34 & $0.01,0.67$ & 0.00 & $-0.05,0.05$ \\
\hline & Q1-Q3 & -7.06 & $-13.74,-0.39$ & 0.31 & $-0.30,0.92$ & 0.23 & $-0.13,0.60$ & 0.48 & $-0.06,1.02$ & 0.01 & $-0.09,0.11$ \\
\hline & Q1-Q4 & -7.37 & $-16.92,2.18$ & 0.17 & $-0.70,1.03$ & 0.16 & $-0.34,0.65$ & 0.43 & $-0.31,1.17$ & 0.01 & $-0.14,0.16$ \\
\hline \multicolumn{12}{|c|}{ Phthalate metabolites ${ }^{\mathrm{e}}$} \\
\hline & Q1-Q2 & -3.44 & $-6.33,-0.55$ & -0.02 & $-0.15,0.11$ & 0.12 & $-0.01,0.25$ & 0.19 & $-0.02,0.41$ & -0.02 & $-0.04,0.01$ \\
\hline & Q1-Q3 & -4.41 & $-8.76,-0.05$ & -0.04 & $-0.31,0.22$ & 0.13 & $-0.07,0.32$ & 0.17 & $-0.14,0.47$ & -0.03 & $-0.09,0.02$ \\
\hline & Q1-Q4 & -2.90 & $-8.35,2.55$ & -0.06 & $-0.46,0.34$ & 0.02 & $-0.23,0.27$ & -0.08 & $-0.45,0.29$ & -0.05 & $-0.13,0.03$ \\
\hline \multicolumn{12}{|l|}{ Boys } \\
\hline & Q1-Q2 & -5.10 & $-9.75,-0.45$ & -0.06 & $-0.25,0.14$ & 0.06 & $-0.16,0.27$ & 0.17 & $-0.15,0.48$ & -0.02 & $-0.06,0.02$ \\
\hline & Q1-Q3 & -6.21 & $-12.94,0.51$ & -0.11 & $-0.50,0.28$ & 0.01 & $-0.31,0.32$ & 0.07 & $-0.38,0.53$ & -0.04 & $-0.12,0.05$ \\
\hline & Q1-Q4 & -3.34 & $-11.29,4.61$ & -0.17 & $-0.75,0.42$ & -0.15 & $-0.55,0.25$ & -0.28 & $-0.88,0.33$ & -0.05 & $-0.18,0.07$ \\
\hline \multicolumn{12}{|l|}{ Girls } \\
\hline & Q1-Q2 & -3.34 & $-7.16,0.49$ & -0.08 & $-0.29,0.13$ & 0.22 & $0.01,0.44$ & 0.21 & $-0.07,0.49$ & 0.00 & $-0.03,0.04$ \\
\hline & Q1-Q3 & -4.46 & $-10.33,1.41$ & -0.16 & $-0.57,0.25$ & 0.32 & $0.01,0.62$ & 0.22 & $-0.19,0.63$ & 0.01 & $-0.06,0.08$ \\
\hline & Q1-Q4 & -3.38 & $-11.17,4.40$ & -0.24 & $-0.86,0.38$ & 0.28 & $-0.08,0.64$ & 0.04 & $-0.48,0.57$ & 0.01 & $-0.10,0.12$ \\
\hline \multicolumn{12}{|l|}{ Bisphenol $^{\mathrm{f}}$} \\
\hline & Q1-Q2 & 0.42 & $-0.63,1.47$ & -0.01 & $-0.08,0.07$ & -0.04 & $-0.09,0.01$ & -0.04 & $-0.11,0.03$ & 0.00 & $-0.02,0.01$ \\
\hline & Q1-Q3 & 0.84 & $-1.26,2.95$ & -0.01 & $-0.17,0.14$ & -0.08 & $-0.19,0.02$ & -0.08 & $-0.22,0.06$ & -0.01 & $-0.04,0.02$ \\
\hline & Q1-Q4 & 1.26 & $-1.89,4.42$ & -0.02 & $-0.25,0.21$ & -0.13 & $-0.28,0.03$ & -0.12 & $-0.33,0.10$ & -0.01 & $-0.06,0.04$ \\
\hline \multicolumn{12}{|l|}{ Boys } \\
\hline & Q1-Q2 & 0.61 & $-0.96,2.17$ & -0.03 & $-0.14,0.07$ & -0.07 & $-0.15,0.00$ & -0.12 & $-0.22,-0.02$ & -0.02 & $-0.04,0.00$ \\
\hline & Q1-Q3 & 1.22 & $-1.92,4.35$ & -0.07 & $-0.28,0.14$ & -0.14 & $-0.29,0.00$ & -0.24 & $-0.44,-0.04$ & -0.04 & $-0.08,0.00$ \\
\hline & Q1-Q4 & 1.83 & $-2.87,6.52$ & -0.10 & $-0.41,0.21$ & -0.22 & $-0.44,0.00$ & -0.36 & $-0.66,-0.06$ & -0.06 & $-0.12,0.01$ \\
\hline \multicolumn{12}{|l|}{ Girls } \\
\hline & Q1-Q2 & -0.08 & $-1.54,1.39$ & 0.07 & $-0.05,0.18$ & -0.01 & $-0.09,0.07$ & 0.07 & $-0.05,0.18$ & 0.00 & $-0.02,0.03$ \\
\hline & Q1-Q3 & -0.15 & $-3.07,2.77$ & 0.14 & $-0.09,0.37$ & -0.02 & $-0.17,0.13$ & 0.13 & $-0.10,0.36$ & 0.01 & $-0.04,0.05$ \\
\hline & Q1-Q4 & -0.23 & $-4.61,4.16$ & 0.21 & $-0.14,0.55$ & -0.03 & $-0.26,0.20$ & 0.20 & $-0.15,0.54$ & 0.01 & $-0.06,0.08$ \\
\hline \multicolumn{12}{|c|}{ OP pesticide metabolites $\mathrm{g}$} \\
\hline & Q1-Q2 & -0.99 & $-2.27,0.29$ & 0.19 & $-0.02,0.39$ & -0.03 & $-0.11,0.04$ & 0.03 & $-0.08,0.14$ & 0.00 & $-0.03,0.02$ \\
\hline & Q1-Q3 & -1.98 & $-4.55,0.58$ & 0.22 & $-0.07,0.50$ & -0.06 & $-0.22,0.09$ & 0.07 & $-0.15,0.28$ & -0.01 & $-0.05,0.04$ \\
\hline & Q1-Q4 & -2.97 & $-6.82,0.88$ & 0.09 & $-0.23,0.42$ & -0.10 & $-0.32,0.13$ & 0.10 & $-0.23,0.43$ & -0.01 & $-0.08,0.06$ \\
\hline \multicolumn{12}{|l|}{ Boys } \\
\hline & Q1-Q2 & -0.81 & $-2.91,1.29$ & 0.12 & $-0.18,0.43$ & -0.01 & $-0.12,0.11$ & -0.02 & $-0.19,0.15$ & -0.01 & $-0.04,0.03$ \\
\hline & Q1-Q3 & -1.62 & $-5.83,2.58$ & 0.14 & $-0.29,0.56$ & -0.01 & $-0.25,0.22$ & -0.03 & $-0.37,0.31$ & -0.01 & $-0.07,0.05$ \\
\hline & Q1-Q4 & -2.44 & $-8.74,3.87$ & 0.05 & $-0.45,0.55$ & -0.02 & $-0.37,0.33$ & -0.05 & $-0.56,0.46$ & -0.02 & $-0.11,0.08$ \\
\hline
\end{tabular}




$\begin{array}{lllllllllll}\text { Q1-Q2 } & -0.99 & -3.01,1.04 & 0.24 & -0.03,0.52 & -0.05 & -0.16,0.06 & 0.05 & -0.13,0.22 & -0.01 & -0.04,0.02 \\ \text { Q1-Q3 } & -1.97 & -6.01,2.07 & 0.28 & -0.09,0.64 & -0.10 & -0.32,0.11 & 0.10 & -0.26,0.45 & -0.03 & -0.08,0.03 \\ \text { Q1-Q4 } & -2.96 & -9.02,3.11 & 0.10 & -0.34,0.54 & -0.16 & -0.48,0.17 & 0.14 & -0.38,0.67 & -0.04 & -0.12,0.05\end{array}$

See Table S1 for biomarker abbreviations.

a Models adjusted for fetal sex (categorical), maternal age (continuous), pre-pregnancy weight (continuous), height (continuous), education level (categorical), ethnicity (categorical), income (categorical), marital status (categorical), parity (categorical), smoking (categorical), alcohol use (categorical), folic acid use (categorical), and child age at assessment (continuous)

b. Sex stratified models adjusted for maternal age (continuous), pre-pregnancy weight (continuous), height (continuous), education level (categorical), ethnicity (categorical), income (categorical), marital status (categorical), parity (categorical), smoking (categorical), alcohol use (categorical), folic acid use (categorical), and child age at assessment (continuous).

c. The inclusion of a quadratic term if improved model fit based on significantly lower Akaike information criteria $($ AIC $)$ : nonverbal IQ $=$ MMP, internalizing problems $=$ DETP, attention problems $=$ MEHHP, aggressive behavior $=$ MCPP.

d. Square root transformed and additionally adjusted for maternal psychopathology.

e. Models of the phthalate metabolite mixture (MMP, MEP, MCPP, MiBP, MnBP, MECPP, MCMHP, MBzP, PA, MEOHP, MEHHP) adjusted for covariates in the total mixtures model plus log 10-transformed pregnancy-averaged concentrations of bisphenol A (BPA) and OP pesticide metabolites (DMDTP, DMTP, DMP, DETP, DEP).

f. Models of the bisphenol mixture (BPA) adjusted for covariates in the total mixtures model plus log 10-transformed pregnancy-averaged concentrations of phthalate (MMP, MEP, MCPP, MiBP, MnBP, MECPP, MCMHP, MBzP, PA, MEOHP, MEHHP) and OP pesticide metabolites (DMDTP, DMTP, DMP, DETP, DEP).

g. Models of the OP pesticide metabolite mixture (DMDTP, DMTP, DMP, DETP, DEP) adjusted for covariates in the total mixtures model plus log 10-transformed pregnancy-averaged concentrations of phthalate metabolites (MMP, MEP, MCPP, MiBP, MnBP, MECPP, MCMHP, MBzP, PA, MEOHP, MEHHP) and bisphenol A. 